\title{
A Centrifuge for All Reasons: Thermo Electron Corporation's extensive range of centrifuges cover all your requirements
}

\author{
Kesava Nagar-Anthal Ph.D \\ Worldwide Product Manager - Floor Model Centrifuges, Thermo Electron Corporation
}

\begin{abstract}
Many processes within the laboratory require the use of at least one centrifuge. For example, a small PCR or DNA modification reaction may require a five second 'spin down' on a simple fixed rotor desktop instrument. Separating cell components on the other hand may require a large, refrigerated, centrifuge with a swing-out rotor. Between these two extremes is a plethora of small, medium and large centrifuges, all designed to meet the specific needs of growing markets.
\end{abstract}

\section{INTRODUCTION}

Choosing a centrifuge certainly isn't an easy task, there are many different parameters to consider such as the need for a refrigerated, ambient or heated chamber; a fixed angle, swing-out, vertical or continuous flow rotor; high, medium or low relative centrifugal force (RCF). Finding the correct centrifuge is therefore an exacting process and very much dependant on the needs of the laboratory or department. With its recent acquisition of Kendro Laboratory Products, Thermo has further expanded its extensive range of centrifuges. Thermo can supply everything needed for a wide range of markets in applications such as: general pelleting; clinical and blood banking; microbiology; tissue culture; molecular biology and genomics; drug discovery; biochemistry and proteomics, as well as nanotechnology.

\section{LIFE SCIENCE APPLICATIONS}

Research today happens at an extremely fast pace with many novel techniques rapidly become accepted standard procedures. As with established protocols, many if not most of these require the use of a range of different centrifuges. For example, it may be necessary to extract a precise membrane bound compartment from a specific cell type. This would involve the use of a refrigerated benchtop or floor standing centrifuge (eg Sorvall Legend and RC-4 respectively), to separate the correct cells from the rest of the matrix, with the exact centrifuge dependant on the cell type and protocol. Once isolated, fractionation of the components from these cells would require a long, density-gradient centrifugation using a refrigerated floor standing ultra-centrifuge, such as the Sorvall Discovery ${ }^{\mathrm{TM}}$ SE models. Extracting molecules such as DNA, RNA or Proteins from these compartments can then be carried-out using standard benchtop procedures involving microcentrifuges, the IEC Micromax and Heraeus ${ }^{\circledR}$ Biofuge ${ }^{\circledR}$ ranges for instance. Downstream of this, reagent 'spin downs' etc, can be completed using these centrifuges and the smaller Jouan B4i/C3i series.

\section{CLINICALLY SPEAKING}

Many clinical diagnostic tests are based on processes originally developed for life science research but with much higher throughput and capacity requirements. Once again the centrifuge used for each task depends on the requirements, but often needs an improved specification due to the nature of the samples and increased throughput. For example, in the pathology departments
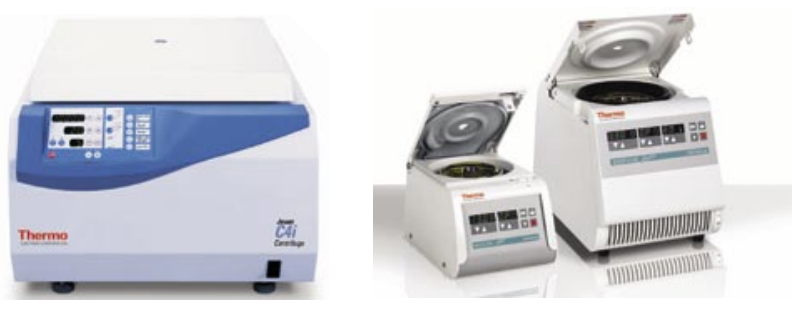

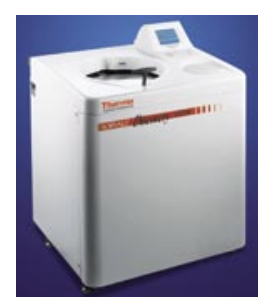

of large hospitals, centrifuges are often in constant use processing large numbers of potentially infectious samples in many different vessels. For this reason, the components need to be highly engineered and quickly interchangeable as well as combining to meet exacting biological safety control standards. Thermo offers a wide range of increased capacity and high throughput centrifuges capable of fulfilling all bio-containment requirements, e.g. Jouan C3i, C4i/G4i and KR4i, as well as the Sorvall RC12BP and Labofuge ranges.

\section{TECHNICAL ADVANCES}

Centrifuges are integral to the majority of research and clinical investigations, but a wide range of factors need to be considered in their design. The changing demands placed upon centrifuges require the constant advancement of the technology used, which not only applies to the physical process itself, but also to the safety features incorporated. Thermo's ultracentrifuges are capable of speeds up to $150,000 \mathrm{rpm}$ where forces reach in excess of $1,000,000 \mathrm{x} \mathrm{g}$. These ultracentrifuges are also increasingly being used in the nanotechnology industry, where very high centrifugal forces are required to separate nano-particles. Therefore lids and catches need to be as secure as possible in case of rotor failure and the controls need to be able to prevent any potentially dangerous settings being used. Traceability is also of growing importance, so Thermo centrifuges can store the information required. Thermo's new software packages and network interfaces also enhance the integration of centrifuges both within the laboratory and between remote locations. Moreover, the introduction of carbon fiber components and new easy-release catches, have not only increased the lifespan of the centrifuge motors, but also made it easier to change between rotors.

\section{CONCLUSIONS}

Thermo has invested heavily in centrifuge research, which has kept them in a world leading position across the whole centrifuge range. The combined Thermo product brands - Heraeus, Sorvall, IEC and Jouan - have been built to address the needs of the users and represent the most advanced centrifuges on the market. The complete Thermo range of centrifuges can be used to cover any possible centrifuge requirements, be they for standard benchtop 'spin downs' or complex high speed separations.

Address correspondence for further information to:

US: Tara Tereso,Tara.tereso@thermo.com

Tel: +1 508-482-7065

EU: Alison Hebbes, Alison.hebbes@thermo.com

Tel: +44 870-609-9203

www.thermo.com/centrifuge 